behaves much less like an organism than a differentiated product of altered cell metabolism.

KENNETH M. SMITH

Plant Virus Research Unit N. XFROS

(Agricultural Research Council), Molteno Institute,

Cambridge.

Sept. 1.

${ }^{1}$ Smith, Kenneth M., Wyckoff, R. W. G., and Xeros, N., Parasit., 42, 287 (1953).

\section{Growth Cycle of Ectromelia Virus in Mouse Liver}

MaNY workers have found that the multiplication of viruses occurs in definite cycles, each involving several stages, namely, adsorption and entry to host cell, a loss of part or all of the infectivity, a 'lag' phase during which no new infective virus can be demonstrated, a period of intracellular rise in infectivity, and finally release of this virus followed by invasion of a fresh population of susceptible cells. We have studied the intracellular multiplication of ectromelia virus in mouse liver, using intravenous inoculation and assaying the infective titre of the liver at intervals. We have found that over a range of inocula from $10^{6}$ to $10^{10} L D 50$ about 30 per cent of the inoculum is adsorbed to the liver in five minutes. Inocula of $10^{\circ} L D 50$ infect practically all the liver cells within five minutes, as measured by a histological method'.

We have found that the multiplication of ectromelia virus also occurs in definite stages. After adsorption there is a rapid fall in infectivity to less than 1 per cent of the initial (five-minute) titre, then a lag period, followed by a rise back to the five-minute titre. Next there is a period of little multiplication followed by a rise in titre, then this last cycle is repeated. This is illustrated in Fig. 1, in which each point represents a pool of two or three mice. When two or more points on a growth curve are obtained from a single mouse the same stepwise rise in titre is found. Such growth curves are obtained with an average dose of one or more $L D 50$ per liver cell, so infection of previously uninfected cells would be quite insufficient to account for the final rise in titre. It appears that multiplication takes place at some intracellular site and the infectious units so produced initiate multiplication on further intracellular sites in the same cell, resulting in the final increase in titre.

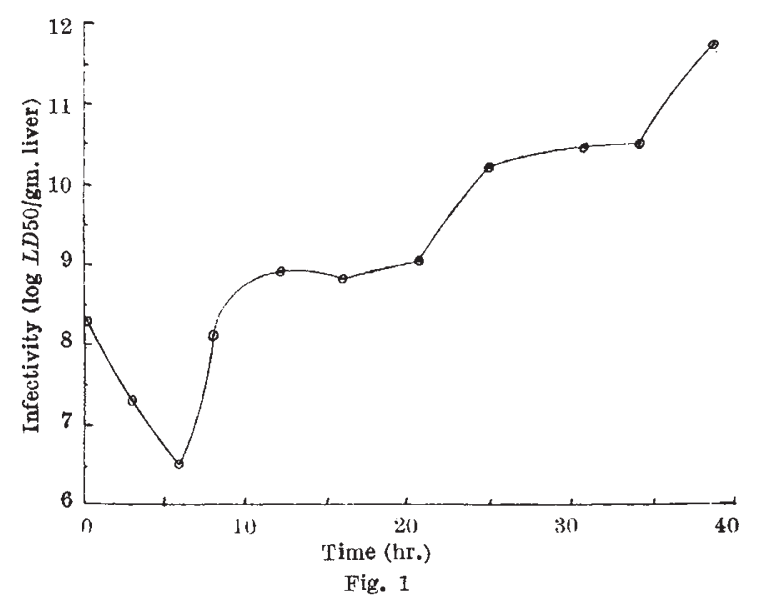

Furthermore, we have been able to infect liver cells with as many as 20 LD50 per cell, assuming $10^{8}$ liver cells per gram of liver. Even with this multiplicity of infection there is no auto-interforence, that is, all twenty particles multiply independently of each other, and reach as high a titre as do lower initial doses.

These two findings would suggest that the fundamental virus-synthesizing centres must be sought at a sub-cellular level.

This work will be reported more fully in a subsequent paper.

G. J. V. Nossax

P. M. DE BURGH

Department of Bacteriology, University of Sydney.

$$
\text { April } 15 .
$$

1 de Burgh, P. M., Aust. J. Exp. Biol. Med. Sci., 28, 213 (1950).

\section{Contribution of Side-Chains to the Infra-Red Spectra of Proteins : the 6.5- $\mu$ Band}

THE strong bands at $3 \mu, 6 \mu$ and $6.5 \mu$ which dominate the absorption spectra of proteins in the fundamental region, and which have been observed as well in $\mathrm{N}$-monosubstituted amides and in synthetic polypeptides, have been attributed to vibrations of the peptide links between the amino-acid residues forming the structural backbone of the protein molecule$^{1}$. It has been established, however, that, in most proteins, side-chains carrying ionizable groups are joined to the polypeptide backbone, and that at the isoionic point the protein exists in the form of dipolar ions. Of the ionizable groups which have been identified, carboxyls occur in the greatest number and may be expected to contribute significantly to the absorption processes in the spectral region of interest.

We have studied the absorption of the carboxyl groups of synthetic polymeric acids and ampholytes in solutions of heavy water (which permit examination of the spectral region $5-8 \mu^{2}$ ) and have found that the band at $5.87 \mu\left(1,703 \mathrm{~cm} .^{-1}\right)$, characteristic of the un-ionized group, disappears upon addition of base, and is replaced by a very intense band at $6 \cdot 40 \mu-6 \cdot 45 \mu\left(1,560-1,550 \mathrm{~cm}^{-1}\right)$, and by another, less prominent one at $7 \cdot 10 \mu\left(1,410 \mathrm{~cm}^{-1}\right)$. These changes in the absorption spectrum, which for poly. methacrylic acid are shown in Figs. $1 A$ and $1 B$, are in agreement with observations on simple fatty and amino-acids, in which the $6 \cdot 4-\mu$ and $7 \cdot 1-\mu$ bands have been attributed to the unsymmetrical and symmetrical valence vibrations of $\mathrm{COO}^{-}$respectively ${ }^{3}$. Although in proteins the $6 \cdot 4-\mu$ band coincides with the strong peptide absorption at $6 \cdot 5 \mu$, it has been possible to establish the contribution of carboxylate groups by working in heavy-water solution, in which the hydrogens in the peptide linkages are completely exchanged for deuterium and the band characteristic of the peptide vibration is shifted to longer wavelengths, probably $6.95 \mu\left(1,440 \mathrm{~cm}^{-1}\right)$. As is evident from Fig. 1C, deuteration of isoionic bovine plasma albumin does not eliminate the absorption in the $6 \cdot 5-\mu$ region-a band remains, centred at $6 \cdot 4 \mu$; upon addition of deuterium chloride to the solution, however, this band disappears and is replaced by a shoulder on the low wave-length side of the $6 \cdot 08 \cdot \mu$ band, as shown in Fig. $1 D$. Simultaneously, the 